\title{
Conus medullaris and cauda equina syndrome as a result of traumatic injuries: management principles
}

\author{
James S. Harrop, M.D., Gabriel E. Hunt Jr., M.D., and AleXander R. Vaccaro, M.D. \\ Department of Neurosurgery, and Department of Orthopaedics, Jefferson Medical College, \\ Rothman Institute, Philadelphia, Pennsylvania
}

\begin{abstract}
Conus medullaris syndrome (CMS) and cauda equina syndrome (CES) are complex neurological disorders that can be manifested through a variety of symptoms. Patients may present with back pain, unilateral or bilateral leg pain, paresthesias and weakness, perineum or saddle anesthesia, and rectal and/or urinary incontinence or dysfunction. Although patients typically present with acute disc herniations, traumatic injuries at the thoracolumbar junction at the terminal portion of the spinal cord and cauda equina are also common. Unfortunately, a precise understanding of the pathophysiology and optimal treatments, including the best timing of surgery, has yet to be elucidated for either traumatic CES or CMS. In this paper the authors review the current literature on traumatic conus medullaris and cauda equina injuries and available treatment options.
\end{abstract}

KEY WorDS • cauda equina syndrome • conus medullaris syndrome $\quad$ trauma

Conus medullaris syndrome and CES are complex neurological disorders manifesting a myriad of symptoms such as back pain, unilateral or bilateral leg pain, paresthesias and weakness, perineum or saddle anesthesia, and rectal and/or urinary incontinence or dysfunction. Patients with CES typically present with symptoms of lumbosacral radiculopathies, whereas those with CMS present with symptoms consistent with spinal cord compression and dysfunction. Both syndromes may occur following traumatic injury to the thoracolumbar junction or the lumbosacral regions.

In a report on intervertebral disc herniation, Mixter and Barr $^{33}$ initially described the relationship between CES and lumbosacral nerve compression as a result of disc herniation. Although CES is clearly related to the presence of an acute large lumbosacral disc herniation, its relationship to acute traumatic injury to the thoracolumbar junction is less clearly defined. Many authors $23,14,17,18,22,27,34,36,40,41$ have defined variable treatment algorithms for the management of CES caused by acute herniation of the nucleus pulposus. Nonetheless, a precise understanding of the pathophysiology and optimal treatments, including the timing of the latter, has yet to be elucidated for either traumatic CES or CMS.

\section{ANATOMY, DISEASE, AND IMAGING}

\section{Anatomical Considerations}

In neonates, the spinal cord terminates at the end of the vertebral column or the lumbosacral junction. Note, how-

\footnotetext{
Abbreviations used in this paper: $\mathrm{CES}=$ cauda equina syndrome; $\mathrm{CMS}=$ conus medullaris syndrome $; \mathrm{CT}=$ computerized tomography; MR = magnetic resonance.
}

ever, that the location of the terminal end of the spinal cord, or the conus medullaris, varies as the infant develops. ${ }^{29}$ During infancy the spinal cord terminates between the first and third lumbar vertebrae, whereas in adults it is positioned between the 12th thoracic and the second lumbar vertebrae. In a detailed anatomical review of the conus medullaris in the adult human, Malas and colleagues ${ }^{29}$ found that the conus had a variable location between $\mathrm{T}-12$ and L-2.

The thoracolumbar region is where the rigid thoracic kyphosis transitions into the mobile lumbar lordosis and is therefore susceptible to traumatic injuries. ${ }^{35}$ This transition generally occurs at T11-12, although in elderly patients with osteopenia the transition point generally migrates caudally due to the increased degree of thoracic kyphosis in this population. ${ }^{4,14,39}$ In the vicinity of the thoracolumbar junction in an adult, the spinal cord terminates as the conus medullaris. Anatomically, this is a dilation of the distal portion of the spinal cord and is the site of transition from the central to peripheral nervous system (cauda equina). This is a very important distinction given the implications for recovery following injury. Patients with an injury above the conus medullaris typically present with symptoms consistent with spinal cord injury, whereas those with injuries below this level may present with symptoms consistent with lumbosacral radiculopathies. Lesions affecting the transition between the two regions can cause symptoms consistent with both upper and lower motor neuron dysfunction.

\section{Natural History of Injury to the Conus Medullaris or Cauda Equina}

Neurological recovery from injury to the conus medullaris or cauda equina is variable and unpredictable and 
may be influenced by many unrelated factors such as patient age, vascular supply to the region, energy transfer to neural elements, pharmacological means to modify the secondary cascade of injury, and timing of neural decompression. Traumatic lesions of the cauda equina, which cause sudden, acute neurological deterioration as opposed to the gradual onset of lower motor neuron dysfunction in chronic CES, generally have a poorer prognosis. ${ }^{27}$

\section{Patient Assessment}

In a minority of patients with a clinically unstable trauma, fractures are not identified immediately during the resuscitative period. It has been reported that between 5 and $15 \%$ of patients with multisystem trauma often have an occult fracture that is not diagnosed during their initial evaluation. ${ }^{9,15,23,28}$ This is particularly pertinent in the context of an unrecognized cauda equina or conus medullaris injury given the potential for a more optimal return of neurological function through directed nonsurgical or surgical intervention. ${ }^{1,27}$ Additionally, symptoms of CES or CMS may be extremely subtle and therefore a heightened degree of suspicion is necessary when encountering traumatic lesions of the thoracolumbar junction so as not to miss an accurate diagnosis. At times, results of the neurological examination may only reveal the presence of mild unilateral perineal anesthesia or urinary retention, findings often missed on routine placement of indwelling catheters during the posttrauma resuscitation portion of a patient's workup. If an unstable fracture is not appropriately immobilized, effects due to repeated trauma to the neural elements may lead to further injury resulting in progressive loss of motor and bowel/bladder function.

\section{Spinal Imaging}

Plain radiographs of the entire spinal axis provide the medical team with an immediate understanding of spinal alignment and the presence of any obvious traumatic disruption of the osteoligamentous anatomy of the vertebral column (Fig. 1). Results positive for trauma on radiography and neurological examination lead to a more focused investigation by using advanced imaging modalities. Computerized tomography scanning provides optimal assessment of bone anatomy and the degree of canal occlusion due to retropulsed bone fragments in the setting of a burst fracture (Fig. 2). ${ }^{12,13}$ Nonetheless, CT scanning has a limited capacity for visualizing the precise size of a traumatic disc herniation, the presence of epidural or subdural hematomas, the nature and degree of ligamentous disruption, or the changes in spinal cord parenchyma. ${ }^{16}$

Magnetic resonance imaging has further improved our ability to visualize and comprehend the degree of soft-tissue ligamentous injury, intravertebral disc disruption and herniation, spinal cord parenchymal edema, and hemorrhage or disruption following spinal injury (Figs. 3 and 4). Magnetic resonance imaging is a noninvasive, nonionizing modality that allows improved visualization of the spinal cord parenchyma and adjacent soft-tissue structures. ${ }^{25}$ Unfortunately, this method is not without its drawbacks: it may not always be available in all institutions at all times, it requires more time to obtain a full complement of images compared with the time taken using other imaging modalities, some patients are uncomfortable with the

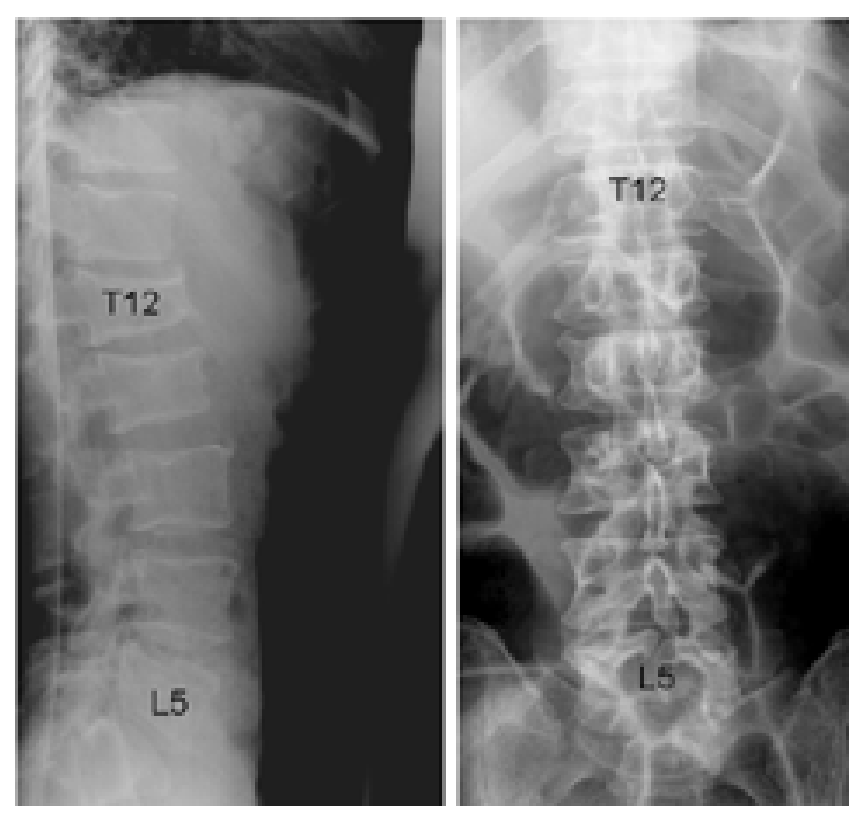

Fig. 1. Lateral (left) and anteroposterior (right) plain radiographs of the thoracolumbar spine obtained following an L-1 flexion compression injury.

process due to the confined imaging space, and patients with specific ferromagnetic implants cannot undergo MR imaging because of the risk of severe soft-tissue injury due to foreign body migration. Findings on MR imaging have been correlated with neurological recovery following trauma; that is, the presence of hemorrhage within the spinal cord parenchyma is associated with minimal neurological recovery. ${ }^{37}$

Note that MR imaging studies are especially useful in

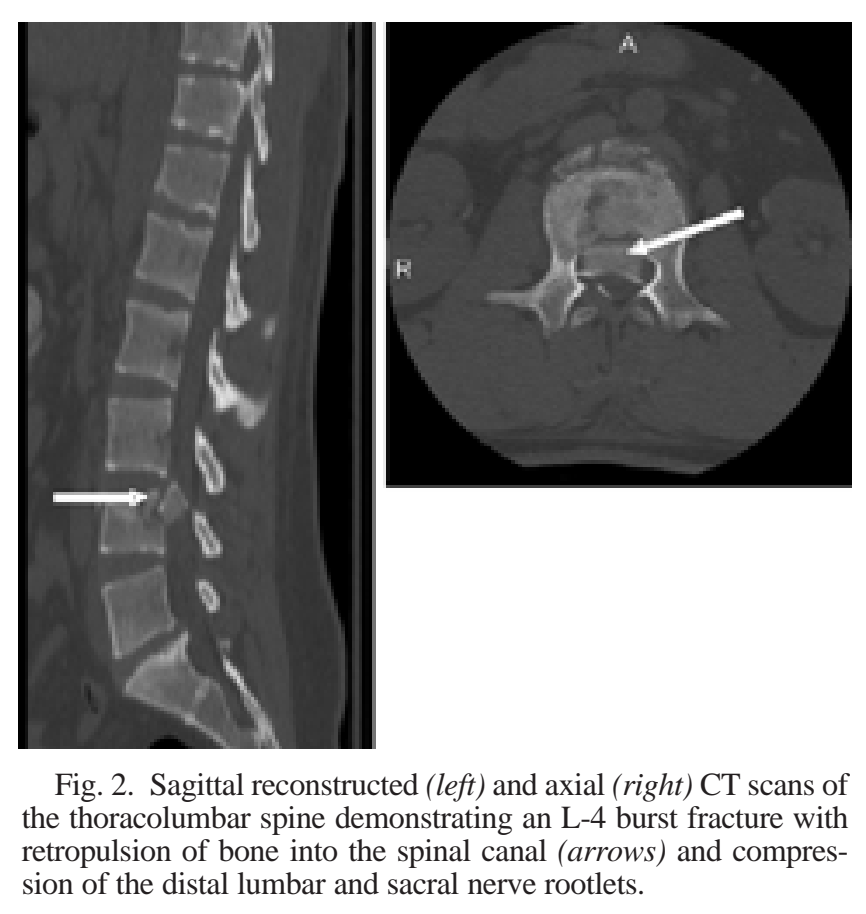




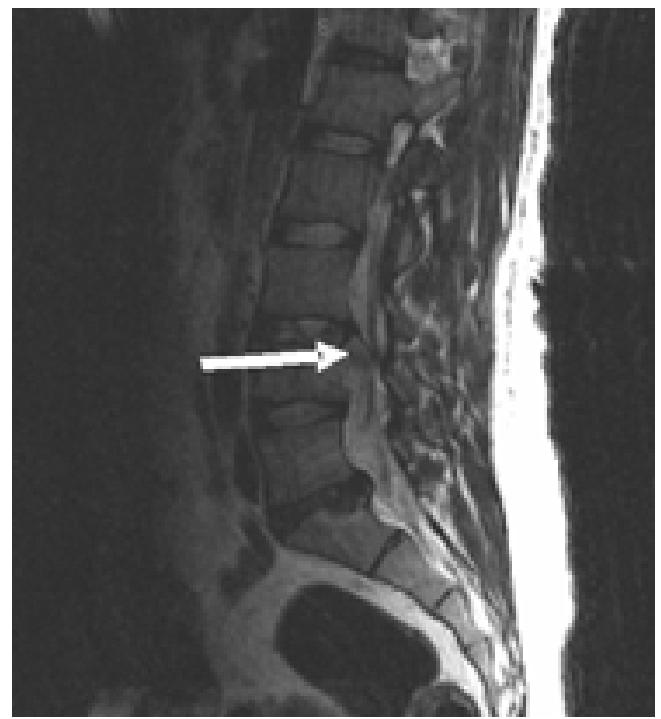

Fig. 3. Magnetic resonance image of the thoracolumbar spine revealing an L-4 burst fracture with retropulsion of bone into the spinal canal (arrow) and compression of the distal lumbar and sacral nerve rootlets.

demonstrating the thoracolumbar junction due to the variable location of the conus medullaris at this level in the adult population..$^{29}$ Patients with neurological dysfunction referable to this region can be difficult precisely to classify neurologically due to the possibility of injury to both the cauda equina and the conus medullaris; the presence of lumbar spinal nerve root sparing and delayed reflex recovery, especially in the setting of drug-induced sedation; and

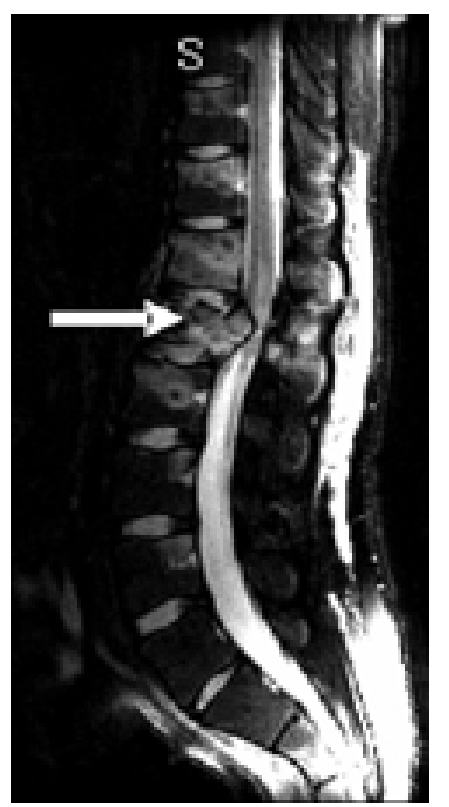

Fig. 4. A $\mathrm{T}_{2}$-weighted $\mathrm{MR}$ image of the thoracolumbar spine demonstrating an L-1 burst fracture with retropulsion of bone into the spinal canal (arrow) and compression of the spinal cord (conus medullaris). Note the increased signal representing cord edema. the presence of an indwelling catheter. Accurate neural visualization may help to clarify the pathophysiology in this clinical situation.

\section{DISEASE MANAGEMENT PRINCIPLES}

The thoracolumbar junction is a spinal region vulnerable to traumatic injury. Mechanisms may include blunt trauma from motor vehicle collisions or penetrating injuries from gunshot or knife injuries. A majority of injuries occurring at this level are either compression or burst fractures. The former type are usually not associated with any neurological deficit given that there is no violation of the spinal canal due to spinal misalignment or retropulsed bone fragments.

Burst fractures by definition involve compromise of the spinal canal by the middle spinal column or spinal misalignment (Fig. 4). These fractures are often managed nonsurgically unless there is significant disruption of the posterior osteoligamentous complex with kyphotic deformity or the presence of a neurological deficit in the setting of significant thecal sac compression. Fractures at the level of the conus medullaris and the cauda equina often are associated with incomplete neurological deficits due to the space available to the spinal cord at these levels.

\section{Penetrating Injuries}

Very few studies have been conducted on the treatment of penetrating traumatic injuries to the cauda equina and conus medullaris. In 1999 Flores, et al. ${ }^{17}$ retrospectively evaluated the neurological outcomes in 45 patients who had incurred gunshot wounds to the spine. Sixty percent of the patients presented with symptoms consistent with CES. Following laminectomy, $53 \%$ of these patients demonstrated improvement in their symptoms. Only a handful of case reports have been published on the onset of CES following stab wounds, the administration of epidural anesthesia, and the spontaneous occurrence of epidural and subdural hematomas from anticoagulation therapy. ${ }^{17,40,41}$ The unifying theme in all of these publications has been the concept of timely diagnosis and surgical decompression in the context of obvious thecal sac compression. Unfortunately, even with timely surgical intervention in these scenarios, functional outcome has often been unpredictable.

\section{Blunt Trauma}

Presently, there is significant controversy concerning the appropriate treatment algorithm in patients who sustain a traumatic injury to either the conus medullaris or cauda equina following blunt trauma. The need for surgery as well as its timing is often a conjectural matter without the definitive support of Class I or II evidence-based literature. No definitive treatment algorithm has been universally accepted for injuries to the conus medullaris or cauda equina despite the number of retrospective reviews on the subject matter. The goal of treatment in such scenarios is to attempt to maximize neurological recovery while preventing further neurological decline and associated pain syndromes.

Once an injury to the conus medullaris or cauda equina has been identified, early intervention is paramount. This 
includes appropriate immobilization of the spinal column and maintenance of physiological vital signs, especially blood pressure and oxygenation. Intravenous administration of methylprednisolone has been advocated by researchers in the National Acute Spinal Cord Injury Study II and III studies in adult patients within 8 hours of spinal cord injury. The effectiveness of this pharmacological regimen has not been uniformly accepted, however. ${ }^{21}$ The efficacy of steroid agents in modifying the secondary cascade of injury to the peripheral nervous system, specifically in CES, has yet to be proven. Gok, et al., ${ }^{20}$ examined the efficacy of methylprednisolone in acute experimental cauda equina injury in a rabbit model. They concluded that both neurophysiological and histopathological study results demonstrated the neuroprotective effectiveness of methylprednisolone if the agent was administered within 8 hours of trauma.

Following hemodynamic stabilization, immobilization, and the decision to administer selected pharmacological agents, the treating physician must decide whether surgical intervention is necessary given the degree of fracture stability. An unstable lesion is fraught with the potential for progressive deformity and pain that may worsen the presenting neurological status in the patient. Surgical intervention is accepted in the setting of significant deformity, that is, kyphosis, or in the presence of a progressive neurological deficit in the setting of a spinal deformity or static neurological compression.

A neurological deficit is present in approximately 4 to $42 \%$ of patients with thoracolumbar junction fractures. ${ }^{18}$ Many researchers believe that neurological damage primarily occurs at the moment of maximal canal occlusion, that is, at the time of injury, and that in the setting of a complete spinal cord lesion, regardless of the degree of residual canal occlusion, surgical decompression rarely, if ever, enhances neurological recovery. ${ }^{5}$ In the event of an incomplete neurological injury with persistent thecal compression, many investigators advocate timely surgical decompression to maximize the potential for neurological recovery of a conus medullaris or cauda equina injury. ${ }^{10}$ Boerger and colleagues ${ }^{5}$ performed a metaanalysis of the world's literature in 2000 to evaluate the effectiveness of surgical decompression in the context of a neurological deficit associated with a thoracolumbar burst fracture. They found that patients with an incomplete neurological deficit who had undergone surgical decompression and stabilization experienced a better neurological recovery compared with that in patients who had undergone nonsurgical treatment. ${ }^{5,7,8,24}$ Surgical intervention also decreased pain, reduced periods of postural reduction or bedrest, and improved sagittal alignment to a greater degree than nonsurgical intervention. ${ }^{19}$ At present, surgical decompression in patients with a complete neurological injury has not demonstrated any real benefit in terms of overall neurological improvement. ${ }^{6}$ Surgery in this context is intended to maximize rehabilitation potential, shorten in-hospital and rehabilitation time, improve spinal stability, and prevent future spinal deformity.

An anterior or posterolateral extracavitary approach may allow for the effective decompression of neural elements in spinal cord compression. The anterior approach is particularly useful in decompressing midline ventral le- sions and correcting severe kyphotic deformities. ${ }^{6,7}$ Bohl$\operatorname{man}^{6}$ reported superior neurological outcomes following an anterior as opposed to a posterior decompressive procedure in patients with an incomplete thoracic spinal cord injury. The major limitation of an anterior exposure is the potential surgical violation of the pleural and/or peritoneal cavities. McCormick ${ }^{30}$ reported the effective use of an anterior extrapleural technique in exposing the thoracic and thoracolumbar spine and avoiding violation of the pleural cavity.

Posterior decompression through a laminectomy following thoracic and thoracolumbar injuries has been shown to be ineffective and should not be performed as an isolated treatment strategy. ${ }^{6,34}$ Surgical removal of the posterior osteoligamentous complex without a concomitant fusion may allow for temporary neurological recovery. Nonetheless, the vertebral column will be unable to maintain its alignment with the loss of its dorsal tension band, and instability together with the potential for loss of spinal alignment may ensue. The immediate result of removing the dorsal osseous components is migration of the spinal cord posteriorly if the spine has a lordotic alignment. Note, however, that normal spinal alignment at the thoracolumbar junction is neutral to slightly kyphotic, which does not allow for optimal thecal sac migration from anteriorly located retropulsed bone fragments. This may result in further neurological compromise due to tethering of the neural elements (bowstring effect) over anterior bone elements.

Advantages of the posterior approach lie in the fact that it provides excellent visualization and access to the dorsal thecal sac. These are useful in managing certain fracture types because the reported incidence of thecal sac lacerations together with possible nerve root incarceration after traumatic thoracic and thoracolumbar burst fractures is between 7 and 16\%. ${ }^{26,38}$ An anterior approach will not provide access to an entrapped lumbar nerve root in this clinical situation. ${ }^{31}$ The presence of a central split in the spinous process or greenstick fracture of the lamina on preoperative transaxial CT studies may be an indicator of a dural laceration, depending on its size and neural displacement during force impact. ${ }^{32}$

The value of timely surgical intervention in a patient with conus medullaris or cauda equina injury following trauma is unclear at this time. Acute surgical intervention in the first 24 to 48 hours after injury, especially by using an anterior approach, is often associated with excessive surgical bleeding, which has motivated many personnel to delay surgical intervention for at least 2 to 3 days following injury. The attempt to maximize neurological recovery through immediate surgical decompression and stabilization has not resulted in clinically improved neurological outcomes compared with outcome following delayed surgical intervention or nonsurgical treatment. In degenerative disease, surgical intervention within 48 hours of symptoms of cauda equina dysfunction has been shown to improve sensory, motor, urinary, and rectal abnormalities significantly compared with intervention after more than 48 hours. ${ }^{3}$ Lesions involving the conus medullaris have a less predictable response to immediate surgical intervention. ${ }^{11}$ Patients with unilateral saddle dysethesias in the setting of CES have a better prognosis for return of blad- 
der function compared with that in patients with bilateral saddle anesthesia. ${ }^{27}$

In summary, the data available to prognosticate the recovery of neurological dysfunction following traumatic conus medullaris or cauda equina injury is derived from retrospective data regarding degenerative disease. $\mathrm{Ob}$ viously, this is far from satisfactory. Today the majority of center personnel who frequently manage traumatic spinal injuries recommend surgical intervention when the patient is medically stable, that is, within the first 3 days of injury, predictably to improve neurological recovery of an incomplete spinal cord or cauda equina injury. More timely intervention is reserved in the event of a progressive neurological deficit with significant thecal sac compression.

\section{References}

1. Ahn UM, Ahn NU, Buchowski JM, et al: Cauda equine syndrome secondary to lumbar disc herniation. Spine 25: $1515-1522,2000$

2. Beaunoyer M, St-Vil D, Lallier M, et al: Abdominal injuries associated with thoraco-lumbar fractures after motor vehicle collision. J Pediatr Surg 36:760-762, 2001

3. Bedbrook GM: Stability of spinal fractures and fracture dislocations. Paraplegia 9:23-32, 1971

4. Besemann EF (ed): The Human Spine in Health and Disease, by Georg Schmorl, ed 2. New York: Grune \& Stratton, 1971, pp 290-291

5. Boerger TO, Limb D, Dickson RA: Does 'canal clearance' affect neurological outcome after thoracolumbar burst fractures? J Bone Joint Surg Br 82:629-635, 2000

6. Bohlman HH: Traumatic fractures of the upper thoracic spine with paralysis. J Bone Joint Surg Am 56:1299, 1974 (Abstract)

7. Bohlman HH, Freehafer A, Dejak J: The results of treatment of acute injuries of the upper thoracic spine with paralysis. J Bone Joint Surg Am 67:360-369, 1985

8. Bradford DS, Akbarnia BA, Winter RB, et al: Surgical stabilization of fracture and fracture dislocations of the thoracic spine. Spine 2:185-196, 1977

9. Chan RN, Ainscow D, Sikorski JM: Diagnostic failures in the multiple injured. J Trauma 20:684-687, 1980

10. Chapman JR, Anderson PA: Thoracolumbar spine fractures and neurologic deficits. Orthop Clin North Am 25:595-612, 1994

11. Chuang TY, Cheng H, Chan RC, et al: Neurourologic findings in patients with traumatic thoracolumbar vertebra junction lesions. Arch Phys Med Rehabil 82:375-379, 2001

12. Dai LY: Remodeling of the spinal canal after thoracolumbar burst fractures. Clin Ortho 382:119-123, 2001

13. de Klerk LW, Fontijne WP, Stijnen T, et al: Spontaneous remodeling of the spinal canal after conservative management of thoracolumbar burst fractures. Spine 23:1057-1060, 1998

14. Dryden DM, Saunders LD, Rowe BH, et al: The epidemiology of traumatic spinal cord injury in Alberta, Canada. Can J Neurol Sci 30:113-121, 2003

15. Enderson BL, Reath DB, Meadows J, et al: The tertiary trauma survey: a prospective study of missed injury. J Trauma 30: 666-670, 1990

16. Flanders AE: Thoracolumbar trauma imaging overview. Instr Course Lect 48:429-431, 1999

17. Flores LP, Nascimento Filho Jde S, Periera Neto A, et al: [Prognostic factors related to gunshot wounds to the spine in patients submitted to laminectomy.] Arq Neuropsiquiatr 57: 836-842, 1999 (Por)

18. Gertzbein SD: Fractures of the Thoracic and Lumbar Spine. Baltimore: Williams \& Wilkins, 1992

19. Gertzbein SD: Scoliosis Research Society. Multicenter spine fracture study. Spine 17:528-540, 1992

20. Gok A, Uk C, Yilmaz M, et al: Efficacy of methylprednisolone in acute experimental cauda equina injury. Acta Neurochir 144:817-821, 2002

21. Hadley MN, Walters BL, Grabb PA, et al: Pharmacological therapy after acute cervical spinal cord injury. Neurosurgery 50 (Suppl):S63-S71, 2002

22. Hu R, Mustard CA, Burns C: Epidemiology of incident spinal fracture in a complete population. Spine 21:492-499, 1996

23. Inaba K, Kirkpatrick AW, Finkelstein J, et al: Blunt abdominal aortic trauma in association with thoracolumbar spine fractures. Injury 32:201-207, 2001

24. Kaneda K, Abumi K, Fujiya M: Burst fractures with neurologic deficits of the thoracolumbar-lumbar spine. Results of anterior decompression and stabilization with anterior instrumentation. Spine 9:788-795, 1984

25. Karnaze MG, Gado MH, Sartor KJ, et al: Comparison of MR and CT myelography in imaging the cervical and thoracic spine. AJR 150:397-403, 1988

26. Keenen TL, Anthony J, Benson DR: Dural tears associated with lumbar burst fractures. J Orthop Trauma 4:243-245, 1990

27. Kostuik JP, Harrington I, Alexander D, et al: Cauda equina syndrome and lumbar disc herniation. J Bone Joint Surg 68: 386-391, 1986

28. Laasonen EM, Kivioja A: Delayed diagnosis of extremity injuries in patients with multiple injuries. J Trauma 31: 257-260, 1991

29. Malas MA, Salbacak A, Buyukmumcu M, et al: An investigation of the conus medullaris termination level during the period of fetal development to adulthood. Kaibogaku Zasshi 76: 453-459, 2001

30. McCormick PC: Retropleural approach to the thoracic and thoracolumbar spine. Neurosurgery 37:908-914, 1995

31. Miller CA, Dewey RC, Hunt WE: Impaction fracture of the lumbar vertebrae with dural tear. J Neurosurg 53:765-771, 1980

32. Miller CA, Hunt WE: Variation of impaction fracture of the lumbar spine. J Neurosurg 56:603, 1982

33. Mixter WJ, Barr JS: Rupture of the intervertebral disc with involvement of the spinal canal. N Engl J Med 211:210-215, 1934

34. Morgan TH, Wharton GW, Austin GN: The results of laminectomy in patients with incomplete spinal cord injuries. Paraplegia 9:14-23, 1971

35. Pick TP, Howden R (eds): Anatomy, Descriptive and Surgical. By Henry Gray. New York: Bounty Books, 1977

36. Rabinovici R, Ovadia P, Mathiak G, et al: Abdominal injuries associated with lumbar spine fractures in blunt trauma. Injury 30:471-474, 1999

37. Schaefer DM, Flanders A, Northrup BE, et al: Magnetic resonance imaging of acute cervical spine trauma. Correlation with severity of neurologic injury. Spine 14:1090-1095, 1989

38. Silvestro C, Francaviglia N, Bragazzi R, et al: On the predictive value of radiological signs for the presence of dural lacerations related to fractures of the lower thoracic or lumbar spine. J Spinal Disord 4:49-53, 1991

39. Singer KP, Jones TJ, Breidahl PD: A Comparison of radiographic and computer-assisted measurements of thoracic and thoracolumbar sagittal curvature. Skeletal Radiol 19:21-26, 1990

40. Tekkok IH, Carter DA, Brinker R: Spinal subdural haematoma as a complication of immediate epidural blood patch. Can J Anaesth 43:306-309, 1996

41. Vapalahti M, Kuurne T: Acute paraplegia caused by a spontaneous extradural haematoma of the conus medullaris area. Acta Chir Scand 141:484-487, 1975

Manuscript received December 29, 2004.

Accepted in final form May 17, 2004.

Address reprint requests to: James Harrop, M.D., 909 Walnut Street, Third Floor, Philadelphia, Pennsylvania 19107. email: James.Harrop@jefferson.edu. 